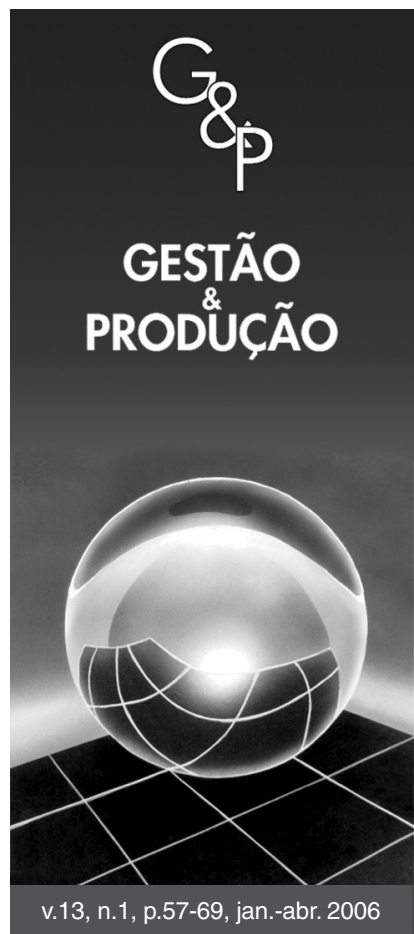

\title{
ANÁLISE BAYESIANA DA CONFIABILIDADE DE PRODUTOS EM DESENVOLVIMENTO
}

\author{
Enrique López Droguett
}

Departamento de Engenharia de Produção, Universidade Federal de Pernambuco, Rua Acadêmico Hélio, s/n, CEP 50740-530, Recife, PE, Brasil, e-mail: ealopez@ufpe.br

\author{
Ali Mosleh \\ Reliability Engineering Program, University of Maryland, \\ 2100 Marie Mount Hall, College Park, MD, USA, \\ e-mail: mosleh@umd.edu
}

Recebido em 17/12/2004

Aceito em 19/1/2006

Resumo

Este artigo apresenta um método para a avaliação da confiabilidade de produtos em desenvolvimento. O método proposto permite a utilização de diversas fontes de informação comumente encontradas nas etapas de desenvolvimento de um produto tais como dados de campo ou dados de garantia (na forma de taxas de falha), dados de teste e evidência subjetiva (opiniões de especialistas com relação ao impacto de modificações de projeto na confiabilidade do produto). Este método também possibilita a incorporação de evidência referente a revisões prévias do mesmo produto ou da mesma informação sobre produtos que são apenas semelhantes ao produto em desenvolvimento. O método proposto é ilustrado pela análise de confiabilidade de tubos de raios X de alta potência, em que se verifica que a avaliação da confiabilidade de um novo projeto antes da execução de testes com unidades, incorporando as modificações de projeto sugeridas, fornece ao fabricante uma relevante fonte de informações para tomadas de decisão referentes à efetiva implementação das modificações de projeto.

Palavras-chave: confiabilidade, produtos em desenvolvimento, teorema de Bayes.

\section{Introdução}

Os consumidores esperam que os produtos adquiridos funcionem como anunciado pelo fabricante. Falhas resultam na insatisfação dos consumidores e elevação de custos com modificações de projeto, processos de fabricação, reparos e subseqüente aumento dos custos de garantia, redução das vendas e danos à imagem corporativa (Hussain, 2003; Priest e Sánchez, 2001; Leech, 1995). Assim, o objetivo do fabricante é desenvolver e comercializar produtos com custos reduzidos e em curtos tempos que satisfaçam as necessidades e expectativas dos consumidores. Pode-se dizer que confiabilidade é um atributo inerente a um produto e que deve ser considerado no seu processo de desenvolvimento. Nas etapas de desenvolvimento de um produto, a estimativa da confiabilidade torna-se um elemento fundamental para a avaliação de desempenho.
A escassez de dados, porém, é uma característica comumente encontrada durante o desenvolvimento de produtos. Esta situação é acarretada por diversas razões, entre as quais:

- Dificuldades em obter dados de falha significativos para o produto sob condições normais de uso;

- Velocidade no avanço tecnológico que requer coleta de dados em curtos períodos de tempo antes que o produto em desenvolvimento se torne obsoleto; $\mathrm{e}$

- Pressões para alcançar prazos e limitações de orçamento.

Desta forma, torna-se imperativo considerar fontes de informação alternativas, tais como testes de vida acelerados e opiniões de especialistas, quando da avaliação da confiabilidade de um produto. São chamados de es- 
pecialistas os engenheiros/projetistas ou outro indivíduo ou grupos de indivíduos familiarizados com o produto e processos objetos de análise.

Diversos têm sido os esforços no sentido de incorporar a avaliação da confiabilidade durante os estágios de desenvolvimento e operação de um produto. Patterson-Hine e Iverson (1990) apresentam um método baseado em árvores de falhas para a avaliação da confiabilidade de sistemas computacionais em aplicações espaciais. Malka e Ziv (1998) discutem a estimação da confiabilidade durante o desenvolvimento da linha de processadores PowerPC da Hewlett-Packard, baseando-se em modelos de confiabilidade de software como o modelo Goel-Okumoto (Goel e Okumoto, 1985), o modelo de Duane (Duane, 1964), e o modelo de Musa-Okumoto (Musa e Okumoto, 1984). Edson e Tian (2004) apresentam um procedimento, para a avaliação da confiabilidade de componentes eletrônicos, baseado no modelo de predição da Telecordia (Telecordia, 2001). Uma abordagem similar é também proposta por Mourad e Fujiwara (2004) no contexto de projetos VLSI. Tian (2005) discute a avaliação da confiabilidade de conversores DC-DC durante o seu processo de desenvolvimento, limitando-se, porém, a dados provenientes de testes acelerados de vida (HALT - Highly Accelerated Life Testing e HASS - Highly Accelerated Stress Screening (Nelson, 1982). Pode-se dizer que esses métodos apresentam como ponto em comum o fato de basearem a estimação da confiabilidade apenas em dados empíricos e provenientes de unidades do produto em desenvolvimento. Não consideram, portanto, a possibilidade de uso de fontes indiretas de informação, como dados parcialmente relevantes de revisões anteriores ou opiniões de especialistas quanto ao impacto das diferenças de projeto entre distintas revisões de um produto. Adicionalmente, os métodos discutidos por Edson e Tian (2004) e Mourad e Fujiwara (2004), ao estimarem as taxas de falha e confiabilidade a partir de modelos baseados em fatores $\pi$ (MIL-HDBK-217-E, 1982), padecem das limitações inerentes a todos os modelos baseados nesses fatores como, por exemplo, falsas suposições de homogeneidade e a não quantificação da incerteza das estimativas de confiabilidade (para uma discussão detalhada sobre o assunto ver Fragola, 1996).

Neste artigo, apresenta-se um método para a estimativa da confiabilidade de um produto em desenvolvimento baseado no teorema de Bayes (De Finetti, 1974; Martz e Waller, 1991). O método proposto permite avaliar a confiabilidade e explicitamente quantificar a incerteza por meio de um processo de quantificação que faz uso das fontes de informação à medida que são disponibilizadas, ou seja, em estágios durante o programa de melhoria da confiabilidade do produto em desenvolvimento. Essas fontes de informação incluem dados de teste realizados pelo fabricante, opiniões emitidas pelos especialistas responsáveis pelo desenvolvimento do produto, e o desempenho observado no campo de unidades com as especificações atuais e com especificações semelhantes.

Uma fonte de informação valiosa corresponde aos dados referentes a projetos anteriores de produtos semelhantes. Apesar destes dados não serem 100\% relevantes, eles fornecem informação valiosa para a melhoria da capacidade preditiva da confiabilidade baseada apenas em dados do produto com as especificações de projeto atuais. Por meio da combinação deste tipo de informação com opiniões de especialistas, severidade de uso em campo, a confiabilidade do produto é estimada.

O método Bayesiano para a avaliação da confiabilidade de produtos em desenvolvimento aqui discutida tem sido encapsulada em uma ferramenta computacional desenvolvida para a Ford Motor Company, a qual é detalhadamente discutida em Groen et al. (2004). Alguns dos elementos do método de análise também têm sido descritos por Lin (2002).

$\mathrm{O}$ artigo está assim organizado. Na próxima seção, é desenvolvido o método de avaliação da confiabilidade para produtos em desenvolvimento. $\mathrm{Na}$ seção 3, é apresentado um exemplo de aplicação a fim de ilustrar a aplicação do método em um caso real. As conclusões são apresentadas na seção 4 .

\section{Estimando a confiabilidade do produto}

O interesse está em estimar-se a confiabilidade de um produto reparável ao longo de seu processo de desenvolvimento. Em outras palavras, deseja-se obter estimativas atualizadas da confiabilidade do produto à medida que novas informações são disponibilizadas. Pode-se tomar como base uma grande população de unidades do produto de tal forma que o impacto de falhas na função intensidade de falha (Rate of Occurence of Failure - ROCOF) (Ascher e Feingold, 1984) do processo estocástico, caracterizando o processo de falha, é mínimo. Assim, assume-se que a função intensidade não é alterada pela ocorrência de falhas. Dado o objetivo principal (avaliação da confiabilidade), o tempo de reparo e métricas de disponibilidade não são de interesse. O processo de falha é, portanto, representado por um processo estocástico pontual no qual se adota a suposição de "tão-bom-quanto-antigo" (as-good-as-old), i.e., um Processo Não-Homogêneo de Poisson é considerado como um modelo apropriado para o processo de falha do produto sob as condições acima consideradas, em que a intensidade de falhas segue um modelo de Weibull. Este modelo é também conhecido como Power Law ou processo de Weibull, pois a intensidade de falha tem a mesma forma funcional da força de mortalidade (ou taxa de falha instantânea) de uma distribuição de Weibull (Rausand e Hoyland, 2004). 
Para um dado intervalo de tempo relativamente pequeno, os dados disponíveis constituem uma população homogênea, ou seja, uma força de mortalidade constante pode ser usada para cada intervalo de tempo. A função intensidade de falha do produto, entretanto, pode variar entre distintos intervalos de tempo, como será mostrado nas seções subseqüentes. Para uma discussão detalhada sobre a avaliação Bayesiana da taxa de falha a partir de populações não-homogêneas, refira-se a Droguett et al. (2004).

A seção 2.1 discute brevemente a distribuição de Weibull e, em seguida, são introduzidos os tipos de informação mais comuns encontrados durante o desenvolvimento de produtos. O desenvolvimento do método é discutido na seção 2.3 e, então, são introduzidos tipos específicos de funções de verossimilhança de acordo com cada tipo de informação considerada.

\subsection{Distribuição de Weibull}

A taxa de falha de um produto que segue esta distribuição é dada pela seguinte expressão (Meeker e Escobar, 1998):

$$
h(t)=\frac{\beta}{\alpha^{\beta}} t^{\beta-1}
$$

em que $\alpha$, e $\beta$ são os parâmetros de escala e forma, respectivamente. Quando $\beta=1$, a taxa de falha é constante. Para $\beta<1$, a taxa de falha é decrescente, e quando $\beta>1$ a taxa de falha é crescente.

Outras estimativas de confiabilidade podem ser obtidas a partir da taxa de falha. Por exemplo, a confiabilidade do produto, $R(t)$, é dada por (Meeker e Escobar, 1998):

$$
R(t)=1-\exp \left[-\left(\frac{t}{\alpha}\right)^{\beta}\right]
$$

\subsection{Tipos de informação para a avaliação da confiabilidade}

O desenvolvimento de um produto é, em geral, um processo evolutivo. Modificações ao projeto original são introduzidas ao longo do período de desenvolvimento do produto, de tal forma que informações obtidas na revisão anterior do projeto podem não ser mais completamente relevantes ao produto, incorporando as mais recentes modificações de projeto. É, em geral, para a revisão atual do produto em desenvolvimento, ou seja, o produto com as mais recentes modificações de projeto, que se está interessado em estimar a confiabilidade. Neste artigo, propõem-se duas categorias para as informações disponíveis para uso na estimativa da confiabilidade da revisão atual do produto:

- Informações sobre o desempenho de revisões anteriores do produto ou mesmo de produtos semelhantes a este. Estas informações serão denominadas de dados de comparação; e
- Informações sobre a revisão atual do produto. Estas informações serão denominadas de dados do produto com revisão atual.

A primeira categoria pode incluir dados de testes (acelerados ou sob condições normais de operação) ou dados de campo. Como discutido a seguir, dados de campo referem-se a informações na forma de taxas de falha obtidas, muitas vezes, pela utilização do produto pelo consumidor. A segunda categoria pode incluir avaliações subjetivas por parte da equipe de projeto sobre o impacto das modificações introduzidas no produto (como mudanças de material, dimensões físicas e parâmetros operacionais), com relação à revisão atual de projeto, dados de testes, sob condições normais ou aceleradas, realizados com o intuito de corroborar ou invalidar as expectativas dos especialistas quanto às modificações adotadas, e dados de campo. A Tabela 1 apresenta um resumo dos diversos tipos de informações.

O objetivo é avaliar a confiabilidade de um produto ao longo de seu desenvolvimento, baseando-se nas informações acima apresentadas. Isto é feito por meio de sucessivas aplicações do teorema de Bayes. Em cada etapa do desenvolvimento do produto, uma categoria de informações é incorporada na avaliação da confiabilidade do produto. A seguir, apresenta-se uma discussão geral do método Bayesiano utilizado.

\subsection{Processo de análise da confiabilidade}

O método implementa um procedimento de análise que divide o problema de avaliação da confiabilidade de um produto em um número de etapas representando estágios no processo evolutivo do projeto do produto. Cada etapa da análise consiste de uma análise Bayesiana, e corresponde a um determinado estágio na evolução prevista para o projeto do produto. Utilizando as fontes de evidências dos tipos descritos na Tabela 1, para cada etapa de análise, obtém-se uma nova estimativa da função de confiabilidade.

Os estágios da análise representando a evolução do produto em desenvolvimento são ilustrados na Figura 1. O primeiro estágio consiste em estabelecer uma avaliação da confiabilidade baseando-se nos dados de comparação. Estes dados geralmente são obtidos de unidades

Tabela 1. Tipos de informações.

\begin{tabular}{ll}
\hline Dados de comparação & \multicolumn{1}{c}{$\begin{array}{c}\text { Dados do produto com } \\
\text { revisão atual }\end{array}$} \\
\hline $\begin{array}{l}\text { Dados de campo } \\
\text { (curvas de taxas de falha) }\end{array}$ & $\begin{array}{l}\text { Impacto das modificações de } \\
\text { projeto (informação subjetiva) }\end{array}$ \\
\hline Dados de testes & $\begin{array}{l}\text { Dados de testes } \\
\text { Dados de campo (curvas de taxas } \\
\text { de falha) }\end{array}$ \\
\hline
\end{tabular}




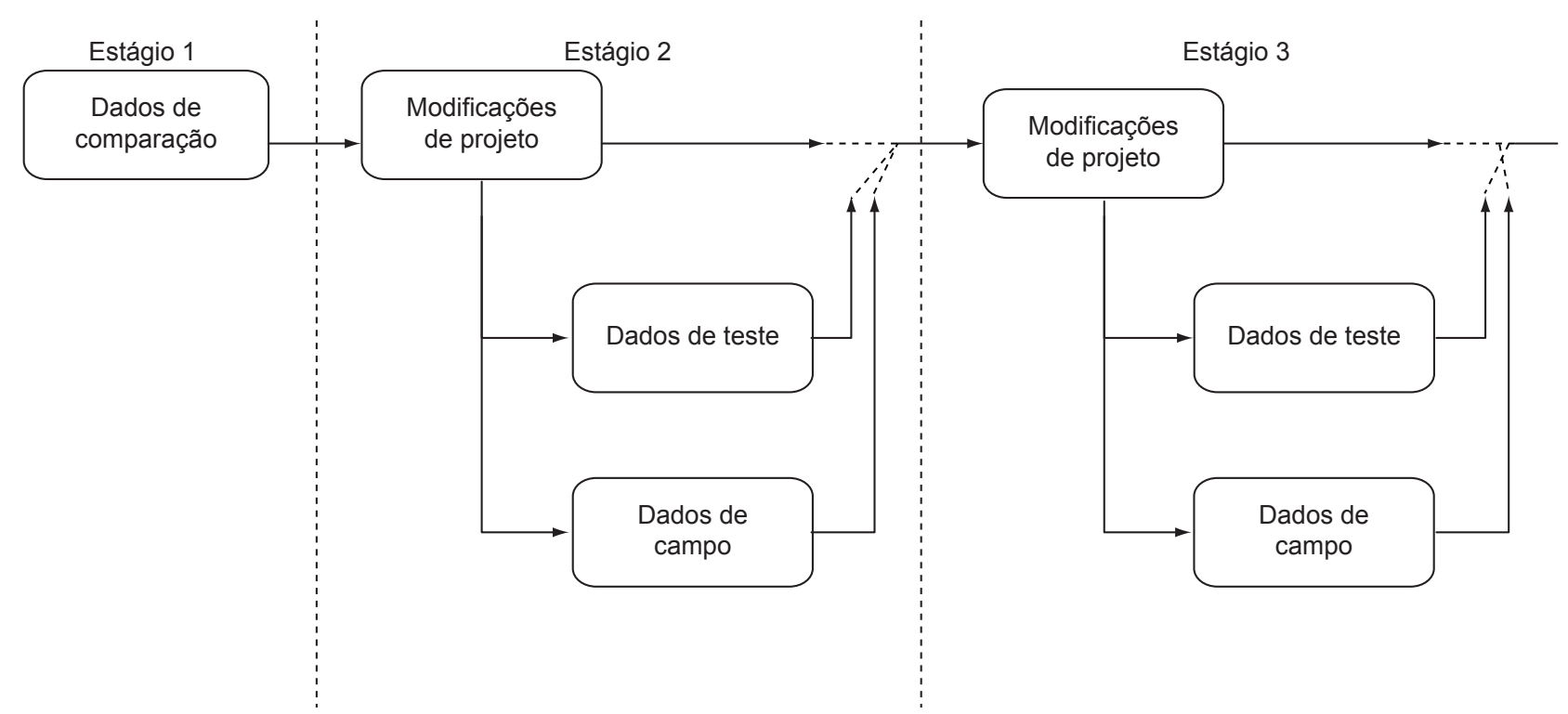

Figura 1. Etapas da análise de confiabilidade.

do produto que já se encontram no mercado, porém com diferenças de projeto com relação ao produto em desenvolvimento. Em seguida, a Figura 1 mostra dois distintos estágios de desenvolvimento, em que são possíveis três etapas de análise dentro de cada estágio. A etapa "Modificações de Projeto" consiste na modificação dos resultados da etapa anterior, baseada nos dados de comparação, e corresponde ao impacto antecipado pela equipe de projeto sobre modificações de projeto propostas pela equipe de desenvolvimento.

A etapa seguinte, denominada "Dados de Teste", é utilizada para validar os resultados obtidos a partir das opiniões dos especialistas sobre o impacto na confiabilidade de alterações de projeto. Nesta etapa, obtém-se uma estimativa atualizada da confiabilidade baseada nos dados anteriores, acrescidos de resultados de testes (acelerados ou sob condições normais de operação) de protótipos do produto com as modificações de projeto propostas. Esta etapa da análise serve, então, para verificar se os dados de teste indicam um comportamento da confiabilidade significativamente diferente do que tinha sido estimado, baseando-se no impacto das modificações de projeto.

A terceira etapa, chamada de "Dados de Campo", corresponde à atualização da confiabilidade baseada também em dados de campo obtidos de unidades da revisão atual do produto (inclusive com as modificações de projeto) em utilização pelos consumidores. A Figura 1 ilustra apenas uma das possíveis configurações de evolução do desenvolvimento de um produto. Diferentes configurações são obtidas, dependendo de quantos estágios de desenvolvimento estejam envolvidos e do tipo de dados disponíveis em cada um desses estágios.

Os resultados são gerados tomando as estimativas de confiabilidade obtidas em uma determinada etapa na evo- lução do projeto do produto como o ponto de partida para a próxima etapa. De uma forma geral, a distribuição a posteriori resultante de uma etapa de análise é atualizada com as novas evidências disponibilizadas para a próxima etapa de análise. Este processo será ilustrado e discutido em detalhes no exemplo de aplicação apresentado na seção 3.

\subsection{Processando as informações}

O interesse está em acompanhar o comportamento da confiabilidade de um produto. Para tanto, é necessário determinar o comportamento da função intensidade de falha e, a partir desta, obterem-se estimativas de outras métricas de confiabilidade, tais como a função de confiabilidade, $R(t)$. Como dito anteriormente, considera-se que a intensidade de falha do produto possui a forma funcional da Weibull dada pela Equação 1, e que toda a informação disponível ao especialista é representada por $E$. Inicialmente, considera-se que o corpo de evidência $E$ corresponde à informação que inclui os tipos acima discutidos ou qualquer combinação destes. Na próxima seção, o desenvolvimento da função de verossimilhança para cada um destes tipos será apresentado.

Uma vez que a intensidade de falha, $h(t)$, é paramétrica, ou seja, $h(t)=h(t \mid \alpha, \beta)$, o problema de estimar $h$ fica reduzido ao de se estimar os parâmetros $\alpha$ e $\beta$, uma vez que cada par $(\alpha, \beta)$ especifica uma única função $h(t \mid \alpha, \beta)$. Desta forma, utiliza-se a evidência disponível $E$ para atualizar o estado de conhecimento sobre os parâmetros de escala e forma da intensidade de falha do produto em questão.

Em outras palavras, obtém-se a distribuição de probabilidade a posteriori sobre $\alpha$, e $\beta$ dado o conjunto de dados representado por $E$, ou seja, $\pi(\alpha, \beta \mid E)$ que representa o estado de conhecimento atualizado sobre os 
parâmetros da intensidade de falha, uma vez que as informações contidas em $E$ foram incorporadas. Assim, aplicando o teorema de Bayes, tem-se:

$$
\pi(\alpha, \beta \mid E)=\frac{L(E \mid \alpha, \beta) \pi_{o}(\alpha, \beta)}{\int_{\alpha} \int_{\beta} L(E \mid \alpha, \beta) \pi_{o}(\alpha, \beta) d \alpha d \beta}
$$

em que $L(E \mid \alpha, \beta)$ e $\pi_{o}(\alpha, \beta)$ são a função de verossimilhança dos dados $E$ e a distribuição a priori dos parâmetros $\alpha$ e $\beta$, respectivamente. Assumindo que a evidência está disponível na forma de $n$ conjuntos de dados independentes, por exemplo, os dados de testes não são correlacionados com os dados de campo, tem-se que a função de verossimilhança pode ser escrita como:

$$
L(E \mid \alpha, \beta)=\prod_{i=1}^{n} L\left(E_{i} \mid \alpha, \beta\right)
$$

em que $L\left(E_{i} \mid \alpha, \beta\right)$ é a função de verossimilhança para um determinado conjunto de dados.

A distribuição a priori, $\pi_{o}(\alpha, \beta)$, deve ser baseada no conhecimento prévio que o especialista possui sobre os parâmetros da intensidade de falha do produto em desenvolvimento. Como se está interessado na avaliação da confiabilidade da revisão atual do produto, considera-se que o analista não possui conhecimento substancial sobre os parâmetros caracterizando o tempo de vida desta mais recente revisão do produto. Isto, entretanto, é suplementado pela utilização de dados de campo de produtos semelhantes ou de revisões anteriores do mesmo produto, considerados de acentuada relevância com relação à revisão atual do produto. Como será discutido nas próximas seções, a informação subjetiva toma basicamente a forma de avaliações sobre a relevância de dados provenientes de produtos semelhantes ou de dados de revisões anteriores à atual revisão do produto, como também sobre a relevância do impacto de modificações de projeto. Desta forma, adota-se uma distribuição a priori constante ('não-informativa'), ou seja, $\pi_{o}(\alpha, \beta)=c$, em que $c$ é uma constante. Para uma discussão sobre a construção de uma distribuição a priori 'informativa' no contexto do modelo de Weibull ver, por exemplo, Nelson (1985), Groen e Droguett (2006).

Uma vez que a distribuição a posteriori dos parâmetros é obtida via Equação 3, pode-se obter uma estimativa atualizada da intensidade de falha do produto. Ou seja, uma estimativa atualizada da intensidade de falha, dado o conjunto de informações $E$. Uma destas estimativas pode ser obtida calculando-se a média sobre todos os valores possíveis dos parâmetros $\alpha, \beta$ :

$$
\bar{h}(t)=\int_{\alpha} \int_{\beta} h(t \mid \alpha, \beta) \pi(\alpha, \beta \mid E) d \alpha d \beta
$$

Por exemplo, no caso considerado, substitui-se a expressão da intensidade de falha com forma funcional equivalente à dada pela Equação 1 na Equação 5, obtendo-se:

$$
\bar{h}(t)=\int_{\alpha} \int_{\beta} \frac{\beta}{\alpha^{\beta}} t^{\beta-1} \pi(\alpha, \beta \mid E) d \alpha d \beta
$$

O mesmo procedimento é utilizado para se obter uma estimativa atualizada da confiabilidade do produto, uma vez que se obtiveram as novas informações $E$ :

$$
\bar{R}(t)=\int_{\alpha} \int_{\beta}\left[1-\exp \left(-\frac{t}{\alpha}\right)^{\beta}\right] \pi(\alpha, \beta \mid E) d \alpha d \beta
$$

Entretanto, é importante notar que para se obterem as estimativas atualizadas da intensidade de falha e da função de confiabilidade é necessário primeiramente avaliar a distribuição a posteriori dos parâmetros de escala e forma, $\pi(\alpha, \beta \mid E)$, por meio da Equação 3. Para tanto, necessita-se especificar as funções de verossimilhança, $L\left(E_{i} \mid \alpha, \beta\right)$, para cada tipo de dado disponível.

Deve-se ressaltar que os resultados numéricos são obtidos por meio de algoritmo Markov Chain Monte Carlo Metropolis-Hastings (Gilks et al., 1996) desenvolvido pelos autores e utilizado para obter uma representação da distribuição conjunta de probabilidade dos parâmetros da intensidade de falha do processo, caracterizando o tempo até a falha do produto (Equação 3). Em seguida, esta distribuição é usada para estimar diversas métricas de confiabilidade e suas respectivas faixas de incerteza, como a confiabilidade e intensidade de falha (Equações 6 e 7, respectivamente).

\subsection{Estimando a confiabilidade: utilizando dados de comparação}

O ponto de partida para estimar a confiabilidade da revisão atual do produto pode ser baseado nos dados de comparação, ou seja, na informação disponível proveniente de revisões anteriores do produto e de produtos semelhantes em desenvolvimento.

Pode haver um ou mais conjuntos de dados de comparação. Como mencionado anteriormente, dados de comparação podem estar disponíveis na forma de dados de campo, dados de teste ou em ambos. Estimativas da intensidade de falha e da confiabilidade do produto podem ser obtidas pela utilização de todas estas fontes de informação. Além do mais, é possível a elucidação e utilização no processo de avaliação da confiabilidade de graus de relevância para cada conjunto de dados de comparação refletindo a aplicabilidade do respectivo conjunto de dados ao produto com revisão atual. A seguir, é apresentada a forma de cada um dos tipos de dados de comparação considerados neste artigo, assim como a respectiva função de verossimilhança.

\subsubsection{Dados de teste}

Este tipo de informação pode estar disponível em duas categorias a depender se foi observada falha do produto durante a realização do teste. 


\subsubsection{Dados de teste que terminaram em falha}

Uma unidade do produto é colocada em teste até que falhe. O tempo de falha, $t_{i}$, desta unidade é a informação relevante. A função de verossimilhança corresponde simplesmente à densidade de probabilidade da distribuição de Weibull avaliada para o instante de falha $t_{i}$ :

$$
L\left(E_{i} \mid \alpha, \beta\right)=\frac{\beta t_{i}^{\beta-1}}{\alpha^{\beta}} \exp \left[-\left(\frac{t_{i}}{\alpha}\right)^{\beta}\right]
$$

\subsubsection{Dados de teste sem a ocorrência de falha}

Uma unidade é colocada em teste por um período predeterminado de tempo. A evidência disponível é o período de tempo, $t_{i}^{*}$, em que a unidade funcionou sem falhas. A função de verossimilhança corresponde à confiabilidade avaliada no instante $t_{i}^{*}$ :

$$
L\left(E_{i} \mid \alpha, \beta\right)=\exp \left[-\left(\frac{t_{i}^{*}}{\alpha}\right)^{\beta}\right]
$$

\subsubsection{Dados de campo}

Também denominado de dados de taxa de falha ou de garantia, este tipo de evidência é composto por curvas de taxa de falha descritas pelo período em funcionamento e a taxa de falha instantânea observada. Para obter-se a função de verossimilhança deste tipo de informação, considera-se que para um dado intervalo de tempo, a taxa de falha instantânea, $h_{i}=h\left(t_{i}\right)$, é constante. Desta forma, pode-se assumir que a probabilidade do número observado de falhas durante cada intervalo de tempo segue uma distribuição de Poisson, ou seja,

$$
P\left(k_{i}, \Delta T_{i} \mid h_{i}\right)=\frac{\left(h_{i} \Delta T_{i}\right)^{k_{i}}}{k_{i} !} e^{k_{i} T_{i}}
$$

em que $k_{i}$ é o número de falhas observado no intervalo de tempo $\Delta T_{i}, h_{i}$ é a taxa de falha instantânea e constante em $\Delta T_{i}$, e $\Delta T_{i}$ é o intervalo de tempo correspondendo às $k_{i}$ observações. Para o caso em estudo, considera-se que a taxa de falha instantânea $h_{i}$ é dada pela distribuição de Weibull (Equação 1). Substituindo esta equação na anterior, a função de verossimilhança para este tipo de informação é dada por:

$$
L\left(k_{i}, t_{i} \mid \alpha, \beta\right)=\frac{\left[\left(\frac{\beta}{\alpha^{\beta}} t_{i}^{\beta-1}\right) \Delta T_{i}\right]^{k_{i}}}{k_{i} !} e^{-\left(\frac{\beta}{\alpha^{\beta}} t^{\beta-1}\right) \Delta T_{i}}
$$

Deve-se ressaltar que dados na forma de taxas de falha instantânea são usados nas situações em que não há acesso direto aos dados brutos (número de falhas e o correspondente intervalo de tempo para uma determinada unidade do produto no campo sob condições normais de uso). Para maiores detalhes sobre a modelagem de diver- sos tipos de dados brutos veja, por exemplo, Droguett et al., 2006; Siu e Kelly, 1998).

\subsection{Utilizando informações parcialmente aplicáveis ao produto: fatores de relevância}

Fatores de relevância são utilizados para indicar o grau de aplicabilidade de conjuntos de dados de comparação, quando utilizados na estimativa da confiabilidade do produto com a revisão atual de projeto. Um determinado fator de relevância reflete o grau de similaridade entre as características de projeto do produto, para o qual os dados foram obtidos, e as da revisão atual do produto. Esta similaridade pode ser obtida não só entre o produto de revisão atual e versões anteriores, mas também com relação a produtos semelhantes (não necessariamente pertencentes ao mesmo produto).

Para este propósito, um número $(0 \leq \omega \leq 1)$ é associado a cada conjunto de dados de comparação como uma medida de relevância. Assim, cada conjunto de dados de comparação, $D C_{i}$, é na realidade um par $\left\{D C_{i}, \omega_{i}\right\}$ em que $\omega_{i}$ é o fator de relevância associado ao $i$-ésimo conjunto de dados $D C_{i}$.

Para a utilização destas informações parcialmente relevantes na estimativa da confiabilidade do produto, pode-se utilizar o Método da Verossimilhança Parcial (MVP) (Groen e Mosleh, 1999). Este procedimento consiste em reduzir o peso de um determinado conjunto de dados de comparação no processo de obtenção da confiabilidade de um produto. Por exemplo, para dois conjuntos de dados de comparação, $D C_{1}$ e $D C_{2}$ com fatores de relevância $\omega_{1}$ e $\omega_{2}$, respectivamente, o MVP resulta em:

$$
L\left(D C_{i}, \omega_{i} \mid \alpha, \beta\right)=\left[L\left(D C_{i} \mid \alpha, \beta\right)\right]^{\omega i} ; i=1,2
$$

em que $L\left(D C_{i}, \omega_{i} \mid \alpha, \beta\right)$ é a função de verossimilhança do $i$-ésimo conjunto de dados parcialmente relevante. Quando $\omega_{1}\left(\right.$ ou $\left.\omega_{2}\right)$ é igual à zero, o conjunto de dados 1 (ou 2) é completamente irrelevante e a função de verossimilhança correspondente é constante. O desenvolvimento a partir deste ponto segue o mesmo procedimento discutido anteriormente.

\subsection{Atualizando a estimativa da confiabilidade do produto}

Até o momento, a confiabilidade do produto foi obtida a partir de fontes de informação de produtos baseados em projetos anteriores e/ou produtos semelhantes. Esta seção discute como atualizar estas estimativas de confiabilidade utilizando dados da revisão atual do produto.

Considera-se que três tipos de informação podem estar disponíveis com relação ao desempenho esperado da revisão atual do produto (veja Tabela 1):

- Impacto das modificações introduzidas no projeto;

- Dados de testes; e 
- Dados de campo.

Os métodos para analisarmos cada uma destas categorias são apresentados a seguir.

\subsubsection{Impacto das modificações de projeto}

Impacto das modificações de projeto corresponde à informação disponível na forma de opiniões de especialistas sobre o tipo e grau do impacto das mudanças introduzidas no projeto do produto com relação à confiabilidade deste, estimada a partir dos dados de comparação. Esta seqüência de análise corresponde a situações tipicamente encontradas no processo de desenvolvimento de novos produtos. Inicia-se com a (quase) completa inexistência de dados referentes à revisão atual do produto e estimativas da confiabilidade são obtidas a partir de dados de campo ou de testes de versões anteriores do produto ou a partir de produtos semelhantes, os quais podem ser completamente ou apenas parcialmente relevantes. Quando da introdução de modificações, os projetistas podem obter estimativas da confiabilidade do novo produto utilizando como fonte de informação as suas opiniões sobre os impactos que estas modificações irão ter na confiabilidade da revisão atual do produto, mesmo antes que protótipos deste estejam disponíveis para teste. Ao existirem tais unidades para teste, a confiabilidade do produto pode ser atualizada pela utilização desta nova informação, sempre se seguindo um processo seqüencial de atualização da confiabilidade via teorema de Bayes.

Podem-se considerar três distintos tipos de impacto decorrentes das modificações de projeto: mudanças na taxa de falha, mudanças no tempo de falha e mudanças nos modos de falha. A magnitude de cada mudança pode ser estimada para diversas etapas durante o tempo de serviço do produto como, por exemplo, para baixa, média e alta quilometragem; ou baixo, médio e alto número de ciclos ou tempo em operação. Cada estimativa, por sua vez, fornecida por um ou mais especialistas, pode ser pessimista, a melhor estimativa ou otimista. Dados desta natureza tipicamente apresentam o formato mostrado na Tabela 2.

Neste artigo, apenas o impacto na taxa de falha do produto será abordado. Assim, a forma apropriada para os impactos na taxa de falha decorrentes das modificações introduzidas é fornecida pelo modelo de perigos proporcionais (Leemis, 1995):

$$
h_{l}(t)=\psi h_{0}(t)
$$

em que $h_{o}(t)$ é a taxa de falha original (antes das mudanças), e $h_{l}$ ( $t$ ) é a taxa de falha alterada e ajustada por um fator de correção $\psi$.

\subsubsection{Impacto dos dados de teste e dados de campo da revisão atual do produto}

As evidências na forma de dados de teste e dados de campo possuem estruturas equivalentes às descritas nas seções anteriores. Os dados de teste são simplesmente incorporados na análise de confiabilidade por meio do uso das funções de verossimilhança fornecidas pelas Equações 8 e 9, enquanto que se usa a Equação 11 para a modelagem dos dados de campo.

\section{Exemplo de aplicação}

Nesta seção, o método é exemplificado pela análise de uma situação real na qual se deseja avaliar a confiabilidade de um tubo de raios $X$ de alta potência para aplicações médicas como mamografias. Este sistema é constituído de quatro subsistemas principais: cátodo, ânodo rotativo, rotor e quadro (revestimento) metálico, como ilustrado na Figura 2. Vamos chamar este sistema de raios $X$ de revisão B. Os dados disponíveis foram obtidos no sistema (para o tubo de raios $\mathrm{X}$ ), não havendo informação respectiva aos subsistemas e seus modos de falha envolvidos nas ocorrências de falha registradas. Desta forma, não será considerada a quantificação da confiabilidade a partir de subsistemas e seus respectivos modos de falha. Quando possível, a análise de confiabilidade de sistemas deve, preferencialmente, ser baseada em dados coletados nos subsistemas, componentes e modos de falha. Desta forma, melhora a qualidade da informação para as tomadas de decisão ao se identificar, por exemplo, o componente ou o modo de falha que maior impacto possui na confiabilidade do sistema, e assim concentrar esforços para a melhoria da confiabilidade desses componentes

Tabela 2. Magnitude da mudança esperada na taxa de falha.

\begin{tabular}{lccc}
\hline & $\begin{array}{c}\text { Baixo tempo } \\
\text { em serviço }\end{array}$ & $\begin{array}{c}\text { Médio tempo } \\
\text { em serviço }\end{array}$ & $\begin{array}{c}\text { Alto tempo } \\
\text { em serviço }\end{array}$ \\
\hline Pessimista & $Y_{B P}$ & $Y_{M P}$ & $Y_{A P}$ \\
Melhor & $Y_{B M}$ & $Y_{M M}$ & $Y_{A M}$ \\
Estimativa & $Y_{B O}$ & $Y_{M O}$ & $Y_{A O}$ \\
Otimista & &
\end{tabular}

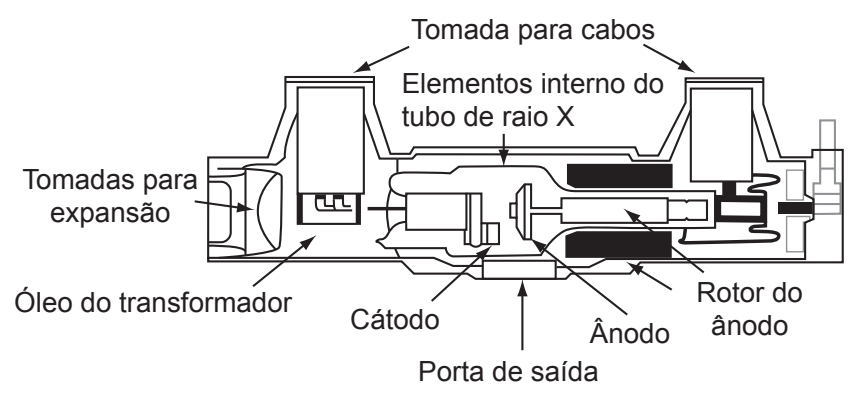

Figura 2. Tubo de raios $\mathrm{X}$ de alta potência com ânodo rotativo. 
específicos durante o processo de desenvolvimento do produto.

Como, em geral, processos de desenvolvimento de produtos são evolutivos ao invés de inovadores, ou seja, na maioria das vezes o projeto de um novo produto baseiase em produtos existentes, considera-se que a revisão A deste produto, incorporando as especificações originais de projeto, tenha sido fabricada e que algumas unidades estejam sendo utilizadas por consumidores. A principal diferença entre esses tubos de raios $\mathrm{X}$ é que a revisão $\mathrm{A}$ possui quadro ou carcaça de vidro, enquanto que a revisão B possui quadro metálico.

Seguindo o fluxo de análise sugerido na Figura 1, o primeiro passo consiste em estabelecer a avaliação de confiabilidade baseada em dados de comparação. Neste exemplo, têm-se dados de campo em termos de taxas de falha instantânea obtidos a partir de unidades de tubos de raios X da revisão A (ver Tabela 3). Os dados foram obtidos pelo fabricante a partir de registros de falha de unidades do produto, durante o período de garantia e em operação em unidades hospitalares nos Estados Unidos. Apesar de dados brutos serem sempre preferíveis, dados de campo na forma de taxas de falha são aqui empregados uma vez que os dados brutos não foram disponibilizados.

Como as duas revisões do produto possuem diferenças de projeto, os dados obtidos para a revisão A não são inteiramente relevantes para a avaliação da confiabilidade de tubos de raios X de revisão B. Desta forma, o analista deve avaliar o grau de similaridade entre as duas revisões, para que os dados sejam apropriadamente incorporados por meio da função de verossimilhança. O grau de similaridade pode ser avaliado em termos, por exemplo, de especificações de projeto, processo de manufatura, e características de aplicação. Como discutido na seção 2.6, a similaridade entre revisões de projeto poder ser avaliada por meio de um fator de relevância obtido a partir das opiniões de especialistas familiarizados com as modificações introduzidas no produto. Se não houver similaridade entre as duas revisões do projeto (o que resulta em fator de relevância nulo), os dados provenientes da revisão anterior serão irrelevantes para a avaliação da confiabilidade da revisão atual do produto. Procedimentos de elucidação e obtenção de fatores de relevância têm sido sugeridos por Droguett et al. (2001), e Firmino et al. (2005), e não serão aqui discutidos. Portanto, supõe-se que o fator de relevância de 0,90 tenha sido obtido, ou seja, a equipe de desenvolvimento acredita que os dados de campo dos tubos de raios $\mathrm{X}$ com revisão A sejam $90 \%$ relevantes quando comparados com a revisão atual (revisão B).

Seguindo o método discutido na seção 2, inicialmente devem-se avaliar os parâmetros de escala e forma por meio da Equação 3. Esta distribuição a posteriori é obtida utilizando-se a função de verossimilhança para os dados de campo em termos de taxas de falha da revisão A mostrados na Tabela 3, Equação 11, e substituindo esta expressão na Equação 11 com $\omega=0,9$ (estamos utilizando o método da verossimilhança parcial). A Figura 3 mostra a distribuição a posteriori dos parâmetros $\alpha$ e $\beta$, representando o que atualmente se sabe sobre estes após obter-se a informação parcialmente relevante do produto com revisão A. O valor mais provável do parâmetro de forma é de aproximadamente $\beta=1,4$, ou seja, o sistema apresenta um processo de deterioração.

Têm-se agora condições de se obter uma estimativa da confiabilidade do tubo de raios $\mathrm{X}$ com revisão $\mathrm{B}$, a partir dos dados de campo parcialmente relevantes de unidades de tubos de raios X de revisão A. De fato, o valor esperado da confiabilidade do sistema é estimado a partir da Equação 7. A Figura 4 mostra a estimativa da confiabilidade do produto correspondendo à mediana (i.e., 50\%), bem como os limites inferior $(5 \%)$ e superior $(95 \%)$ do intervalo de probabilidade. Como mostrado na Figura 4, diferentes percentis para a confiabilidade são também

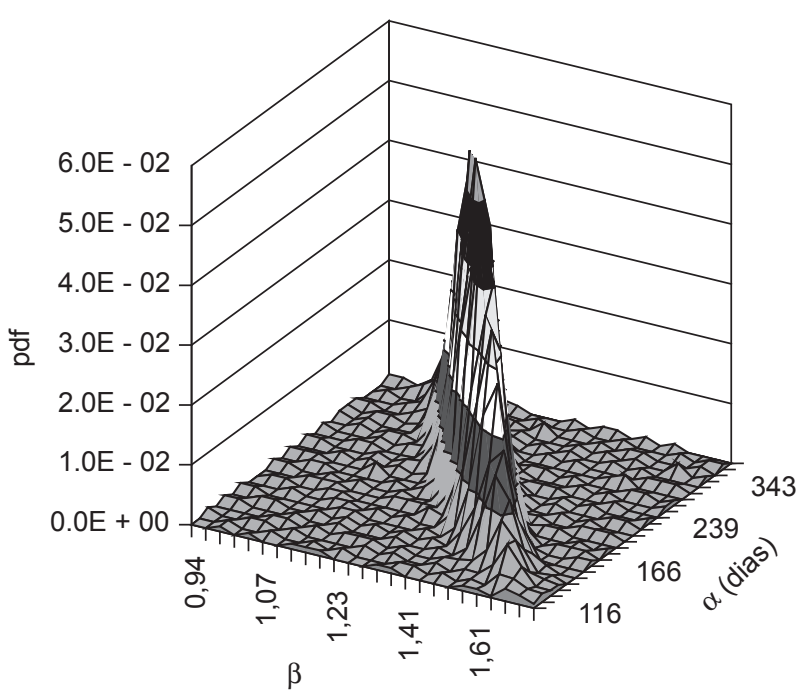

Figura 3. Distribuição a posteriori dos parâmetros da intensidade de falha do tubo de raios $X$ de revisão $B$ baseada nos dados de comparação.

Tabela 3. Dados de campo de tubos de raios $\mathrm{X}$ de revisão A.

\begin{tabular}{ccllllllllllllllll}
\hline Dias em serviço & 1 & 2 & 3 & 4 & 5 & 6 & 7 & 8 & 9 & 10 & 11 & 12 & 13 & 14 & 15 & 16 & 17 \\
\hline Taxa de falha $\left(\mathbf{1 0}^{\mathbf{2}}\right)$ & 1,9 & 2,0 & 1,6 & 1,7 & 1,7 & 1,6 & 1,6 & 2,0 & 2,5 & 2,4 & 2,5 & 2,4 & 2,5 & 3,0 & 2,2 & 2,7 & 1,7 \\
\hline
\end{tabular}


estimados. Desta forma, os resultados fornecem uma quantificação explícita do grau de incerteza em torno da métrica de confiabilidade sendo avaliada.

Depois de estabelecida a avaliação preliminar da confiabilidade do produto na sua revisão $\mathrm{B}$, baseada apenas em dados de campo da revisão $\mathrm{A}$, avalia-se o efeito na confiabilidade do sistema das modificações de projeto introduzidas: mudança do quadro de vidro para quadro metálico. Em geral, como nesta etapa do desenvolvimento de um novo produto, protótipos da revisão B ainda não foram disponibilizados, não há dados de teste que possam ser utilizados na estimativa da confiabilidade. Assim, a única fonte de informação disponível corresponde ao conhecimento e experiência dos próprios especialistas de projeto/confiabilidade.

Desta forma, o impacto das modificações de projeto pode ser inicialmente avaliado em termos da magnitude da mudança esperada na taxa de falha do tubo de raios $\mathrm{X}$ com revisão B. Seguindo o procedimento proposto na seção 2.7, um especialista ou grupo de especialistas estimam valores pessimista, otimista e a melhor estimativa para a magnitude da mudança na taxa de falha do tubo de raios $\mathrm{X}$, quando da incorporação das modificações de projeto sugeridas. Para a presente situação, a elucidação do líder da equipe de desenvolvimento resultou nas seguintes estimativas:

- Otimista: 0,5, i.e., $50 \%$ de redução na taxa de falha do produto com revisão $\mathrm{B}$;

- Melhor estimativa: 0,7, i.e., $30 \%$ de redução na taxa de falha da revisão B; e

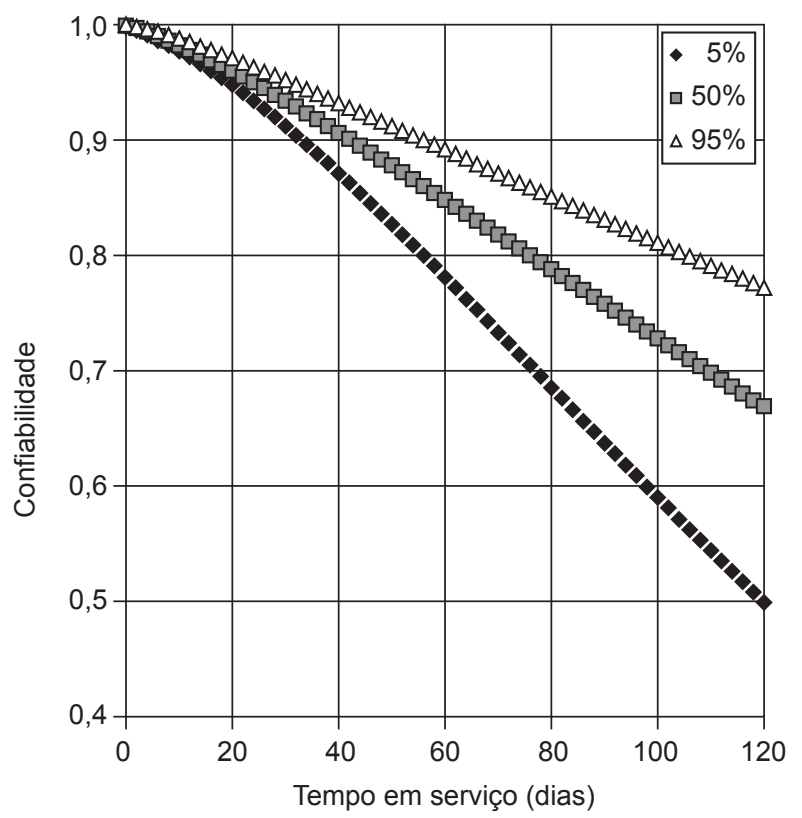

Figura 4. Confiabilidade do tubo de raios $X$ com revisão B estimada a partir dos dados de comparação.
- Pessimista: 1.0, i.e., a taxa de falha do produto permanece inalterada. As modificações propostas não surtem o efeito desejado (redução da taxa de falha).

O especialista não cogita da elevação da taxa de falha após as modificações terem sido introduzidas no produto. Este caso, porém, pode ser contemplado simplesmente utilizando-se um valor maior que 1 . O especialista fornece estimativas referentes ao impacto esperado das modificações de projeto sobre a taxa de falha instantânea e não sobre a intensidade de falha.

Com esta nova fonte subjetiva de informação, podese obter uma nova estimativa da confiabilidade do tubo de raios $\mathrm{X}$ de revisão $\mathrm{B}$ por meio do mesmo processo de quantificação utilizado anteriormente. Agora, porém, a distribuição conjunta de probabilidade dos parâmetros da distribuição Weibull mostrada na Figura 4 é atualizada com a nova fonte de evidência representada pelo ajuste na taxa de falha (Equação 13). De posse desta nova distribuição de probabilidade a posteriori dos parâmetros, estima-se novamente a confiabilidade do produto de Revisão B segundo a Equação 7.

De fato, a Figura 5 mostra tanto a confiabilidade estimada com base apenas nos dados de taxa de falha da revisão A, como também a estimativa atualizada da confiabilidade após ter sido obtida a informação adicional na forma de opiniões do especialista. Com o intuito de simplificar, a Figura 5 mostra apenas as curvas de confiabilidade correspondentes às medianas (i.e., 50\%), omitindo

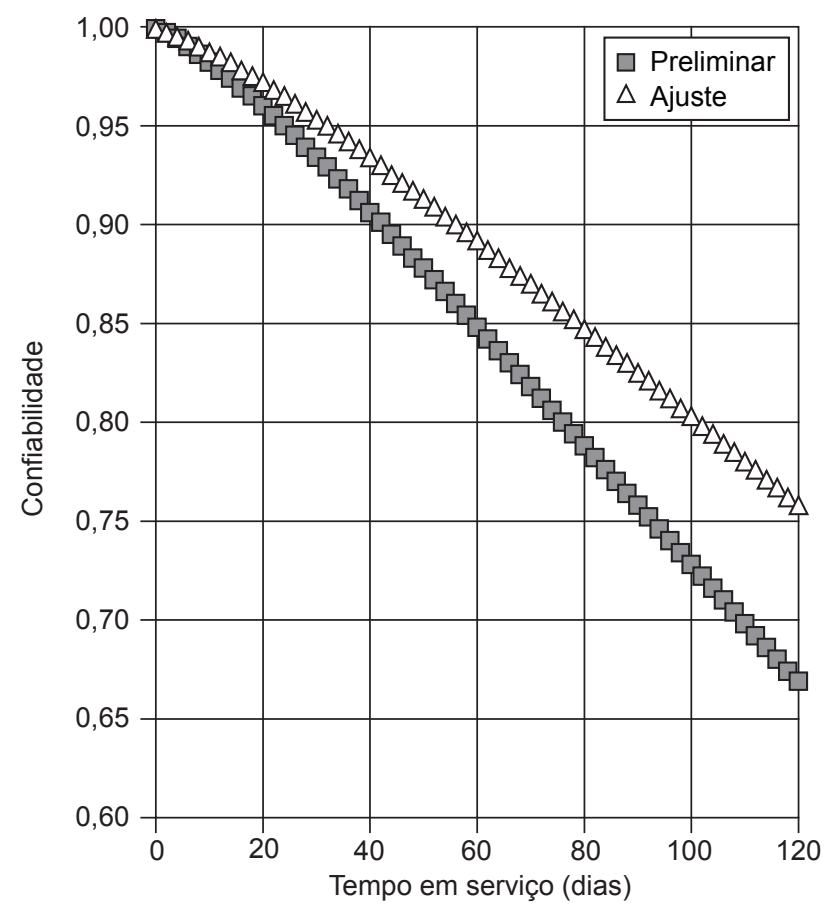

Figura 5. Confiabilidade do tubo de raios $X$ com revisão $B$ antes e após as opiniões do especialista. 
os limites inferior e superior do intervalo de probabilidade. A partir desta figura, pode-se observar que a confiabilidade do sistema melhora com relação à estimativa anterior, resultado este que reflete a expectativa do especialista consultado, manifestada em termos de redução da taxa de falha do tubo de raios X com revisão B. Ou seja, espera-se que as modificações introduzidas levarão de fato a uma melhoria da confiabilidade do sistema. A incerteza em torno das estimativas da confiabilidade pode ser expressa em termos de um intervalo de probabilidade com limites inferior (5\%) e superior (95\%), avaliados para um determinado ponto do tempo. Por exemplo, para $t=10,60,120$ dias, têm-se, respectivamente, os intervalos $(0,977 ; 0,988),(0,781 ; 0,892),(0,499 ; 0,772)$ para a confiabilidade quando apenas considerados os dados de comparação. Ao considerar também as opiniões do especialista, os intervalos de probabilidade com limites inferior (5\%) e superior (95\%) para a confiabilidade são $(0,979 ; 0,996),(0,806 ; 0,958),(0,555 ; 0,904)$ para $t=10$, 60,120 dias, respectivamente. Como as opiniões otimista e melhor estimativa do especialista correspondem a reduções na taxa de falha instantânea do produto, percebe-se que esses intervalos de probabilidade da confiabilidade, após a incorporação das opiniões do especialista, possuem limites inferiores (5\%) e superiores $(95 \%)$ de maior magnitude do que os correspondentes apresentados para a confiabilidade estimada, baseando-se apenas nos dados de comparação.

Entretanto, tais expectativas precisam ser confirmadas. Para isto, a próxima etapa geralmente envolve a obtenção de dados de falha provenientes de testes de unidades do produto com revisão $\mathrm{B}$.

Considerando os dados de testes sob condições nãoaceleradas de unidades do tubo de raios $\mathrm{X}$ de revisão $\mathrm{B}$ mostrados na Tabela 4, para obter-se a nova estimativa da confiabilidade, precisa-se primeiro obter a distribuição dos parâmetros de escala e forma da intensidade de falha dada à nova fonte de evidência. Ou seja, a distribuição $a$ posteriori dos parâmetros da intensidade de falha obtida, baseando-se nas opiniões do especialista é agora usada como ponto de partida para a próxima etapa na qual os dados de testes (Tabela 4) foram disponibilizados. Esta distribuição será atualizada via Equação 3 (teorema de Bayes), resultando em uma nova distribuição de probabilidade a posteriori dos parâmetros.
Alguns testes resultaram em falha, enquanto em outros não foi observada falha da unidade. Desta forma, utilizase a função de verossimilhança dada pela Equação 8 para os dados de testes que terminaram em falha, e a Equação 9 para os testes sem falhas. A distribuição a posteriori dos parâmetros de escala e forma é então calculada a partir da Equação 3. Na Figura 6, observa-se que o valor mais provável do parâmetro de forma é aproximadamente $\beta=1,2$, e o parâmetro de escala é $\alpha=250$ dias. Quando comparados com os valores obtidos a partir apenas dos dados de taxa de falha ( $\beta=1,4$ e $\alpha=215$ dias), os novos resultados indicam que realmente houve uma melhoria na confiabilidade do produto.

De fato, a Figura 7 mostra a nova estimativa da confiabilidade após os dados de testes realizados com unidades da revisão B terem sido obtidos. Para facilitar a comparação, estão mostradas nesta figura as estimativas da confiabilidade obtidas nas três etapas do processo de análise: preliminar (dados de falha), ajuste (com as opiniões do especialista), e testes (resultados das unidades com revisão B). Pode-se observar que os resultados vêm corroborar a expectativa da equipe de desenvolvimento:

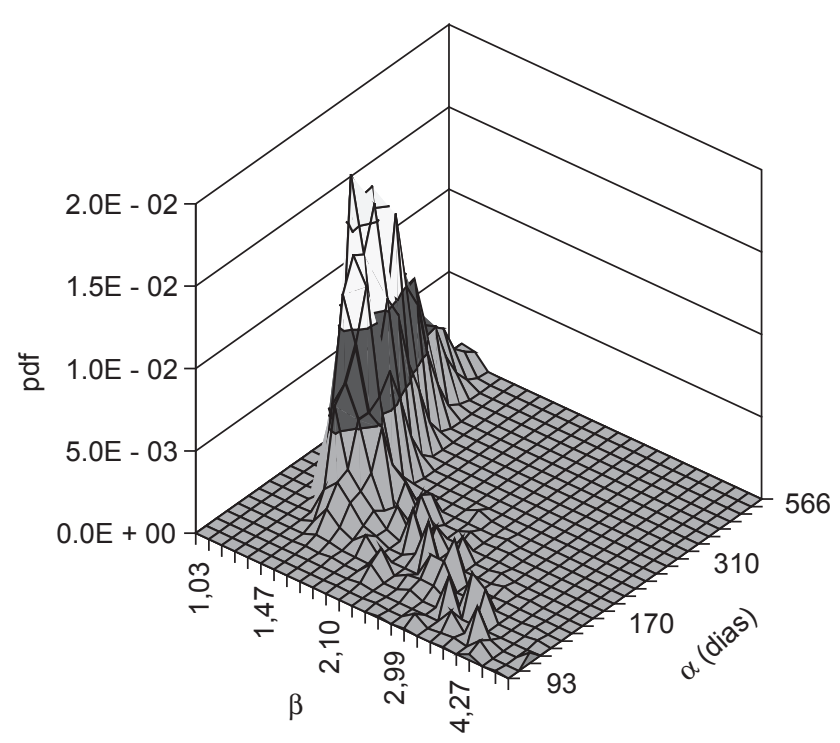

Figura 6. Distribuição a posteriori dos parâmetros da intensidade de falha após incorporar os dados de teste de tubos de raios $X$ com revisão $B$.

Tabela 4. Dados de testes não-acelerados de tubos de raios $\mathrm{X}$ com revisão $\mathrm{B}$.

\begin{tabular}{|c|c|c|c|c|c|c|c|c|}
\hline \multicolumn{9}{|c|}{ Duração do teste (dias) } \\
\hline $73,6^{*}$ & $82,4^{*}$ & $83,2 *$ & $86,4^{*}$ & $90,4^{*}$ & $89,6^{*}$ & $71,2 *$ & $65,6^{*}$ & $98,4^{*}$ \\
\hline $55,2 *$ & $74,4^{*}$ & $80,0^{*}$ & $91,2 *$ & $85,6^{*}$ & $74,4 *$ & $76,8^{*}$ & $56,8^{*}$ & 53,6 \\
\hline 60,8 & 60,0 & 64,0 & $84,8 *$ & $80,0 *$ & $60,8^{*}$ & 52,8 & $78,4^{*}$ & $81,6^{*}$ \\
\hline
\end{tabular}

(*) Indica testes terminados sem a ocorrência de falha. 


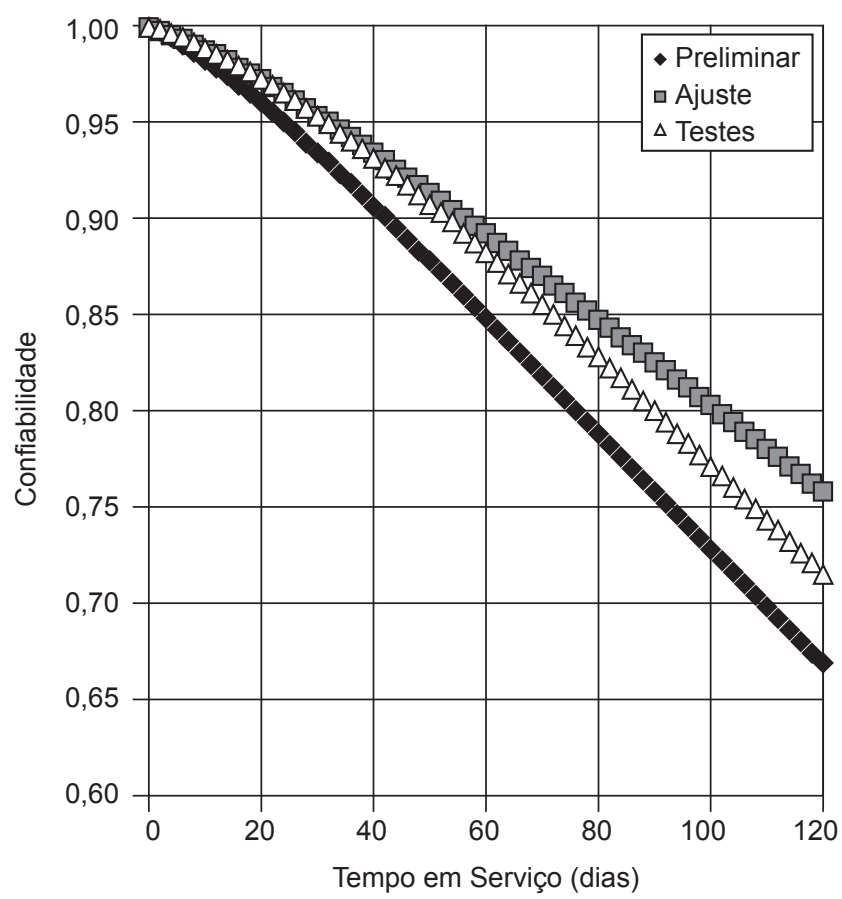

Figura 7. Evolução da confiabilidade do tubo de raios $\mathbf{X}$ com revisão $B$.

as modificações introduzidas acarretaram na melhoria da confiabilidade do produto com relação aos resultados preliminares.

Entretanto, deve-se notar que as opiniões fornecidas pelo especialista foram demasiadamente otimistas quando comparadas à confiabilidade antes e após a obtenção dos dados de testes de unidades incorporando as modificações de projeto, ou seja, a avaliação atualizada da confiabilidade do sistema é inferior à estimativa obtida na etapa anterior baseada nestas opiniões. As modificações impactaram positivamente a confiabilidade do sistema, mas não como antecipado pelo especialista consultado (líder da equipe de desenvolvimento).

As estimativas da confiabilidade mostradas na Figura 7 são obtidas a partir de atualizações sucessivas da distribuição conjunta de probabilidade $a$ posteriori dos parâmetros da intensidade de falha, à medida que novas evidências vão sendo disponibilizadas. O processo de estimação não consiste simplesmente na execução de três aplicações separadas e estanques do teorema de Bayes, representado pela Equação 3. De fato, as três análises Bayesianas são interligadas, uma vez que a distribuição a posteriori da primeira etapa é o ponto de partida para a segunda, a qual é atualizada pelas evidências disponíveis naquela etapa do desenvolvimento do produto, assim, como esta, por sua vez, é o ponto de partida da terceira etapa que é atualizada com as evidências disponibilizadas nesta. O método, portanto, incorpora as evidências à medida que vão sendo disponibilizadas, em um processo de atualização cronológico e em estágios.

\section{Conclusões}

Neste artigo, foi apresentado um método para avaliar a confiabilidade de produtos em desenvolvimento. O método proposto possibilita fazer uso de diversos tipos de informação na obtenção de estimativas de confiabilidade atualizadas, à medida que novos dados são disponibilizados nas diferentes etapas do desenvolvimento de um produto. Em particular, o método foi discutido em termos de dados de campo (taxas de falha instantânea), dados subjetivos na forma de opiniões de especialistas e dados de testes obtidos a partir de unidades do produto em questão.

Característica importante deste método é a flexibilidade em usar informações de revisões anteriores do produto ou provenientes de produtos semelhantes. Estas informações podem ser totalmente ou apenas parcialmente relevantes ao processo de avaliação da confiabilidade do produto em desenvolvimento.

Um exemplo de aplicação foi apresentado para ilustrar a avaliação da confiabilidade e acompanhar a sua evolução ao longo do processo de desenvolvimento do produto. A sequiência na análise da confiabilidade seguida no exemplo, entretanto, não é de forma alguma a única possível. Variações são possíveis dependendo, por exemplo, das características do produto e de quais tipos de dados estão disponíveis nas diferentes etapas do seu desenvolvimento. O procedimento de avaliação da confiabilidade aqui discutido é geral quanto a este aspecto.

O método permite também o desenvolvimento de estimativas de confiabilidade para um novo projeto antes da realização de testes com unidades dessa revisão do produto. Em particular, as estimativas atualizadas da confiabilidade com base nas opiniões de especialistas sobre o impacto de modificações de projeto fornecem ao fabricante uma fonte importante de informações para tomadas de decisão referentes à efetiva implementação das modificações de projeto sugeridas pela equipe de desenvolvimento. Isto beneficia o fabricante em áreas como a alocação de recursos durante o processo de desenvolvimento do produtor. Além do mais, o método possibilita a quantificação explícita da incerteza em torno de uma dada métrica de confiabilidade, auxiliando na tomada de decisões sob elevado nível de incerteza. 
ASCHER, H.; FEINGOLD, H. Repairable systems reliability. Marcel Dekker, New York, 1984.

DEPARTMENT OF DEFENCE. MIL-HDBK-217-E: Reliability prediction of electronic equipment. Washington DC, 1982.

DE FINETTI, B. Theory of Probability. Volumes 1 and 2, John Wiley \& Sons, New York, 1974.

DROGUETT, E. L. et al. Methodology for the treatment of model uncertainty - applications to fire risk models. In: EUROPEAN SAFETY AND RELIABILITY ASSOCIATION CONFERENCE, 2001, Torino, Italy. Proceedings of the European safety and reliability association conference. Torino, Italy, 2001.

DROGUETT, E. L.; GROEN, F.; MOSLEH, A. The combined use of data and expert estimates in population variability analysis. Reliability Engineering \& System Safety, v. 83, n. 3, p. 311-321, 2004.

; Bayesian assessment of the variability of reliability measures. Aceito para publicação na revista Pesquisa Operacional, 2006.

DUANE, J. T. Learning curve approach to reliability monitoring. IEEE Transaction on Aerospace, v. 2, p. 563566, april 1964.

EDSON, B.; TIAN, X. A prediction based design-for-reliability tool. In: ANNUAL RELIABILITY AND MAINTAINABILITY SYMPOSIUM, 26-29 January 2004, Los Angeles, USA, Proceedings of the annual reliability and maintainability symposium, p. 412-417, 2004.

FIRMINO, P. R.; MENÊZES, R. C.; DROGUETT, E. L. Método aprimorado para a quantificação do conhecimento em análises de confiabilidade por redes Bayesianas. In: SIMPÓSIO BRASILEIRO DE PESQUISA OPERACIONAL, SBPO, 37, 27 a 30 de Setembro, Gramado, Brasil, 2005. Anais do SBPO, 37, Gramado, 2005.

FRAGOLA, J. R. Reliability and risk analysis data base development: an historical perspective. Reliability Engineering and System Safety, v. 51, n. 2, p. 125-136, 1996.

GILKS,W.R.;RICHARDSON,S.;SPIEGELHALTER, D. J. Markov Chain Monte Carlo in Practice. Chapman and Hall, 1996.

GOEL, A. L.; OKUMOTO, K. Time-dependent error detection rate model for software and other performance measure. IEEE Transactions on Software Engineering, v. 11, n. 12, p. 385-306, 1985.

GROEN, F.; MOSLEH, A. Behavior of weighted likelihood and weighted posterior methods for treatment of uncertain data. In: EUROPEAN CONFERENCE ON SAFETY
AND RELIABILITY, Munich, Germany, 1999. Proceedings of the European safety and reliability association conference, Munich, Germany, 1999.

GROEN, F.; DROGUETT, E. L.; JIANG, S.; MOSLEH, A. A reliability data collection and analysis system for products under development. Brazilian Journal of Operations \& Production Management, v. 1, n. 1, 2004.

GROEN, F.; DROGUETT, E. L. Prior specification for multi-failure mode Weibull reliability models. In: ANNUAL RELIABILITY AND MAINTAINABILITY SYMPOSIUM. Newport Beach, California, 2006. Proceedings of the annual reliability and maintainability symposium, 2006.

HUSSAIN, A. Z. M. O.; MURTHY, D. N. P. Warranty and optimal reliability improvement through product development. Mathematical and Computer Modelling, v. 38, n. 11-13, p. 1211-1217, 2003.

LEECH, D. J. Proof of Designed Reliability. Engineering Management Journal, v. 5, n. 4, p. 169-174, 1995.

LEEMIS, L. M. Reliability: Probabilistic Models and Statistical Methods. Prentice-Hall, New Jersey, 1995.

LIN, J. C. The use of indirect evidence for Bayesian reliability analysis. In: ANNUAL RELIABILITY AND MAINTAINABILITY SYMPOSIUM, Seattle, Washington, 2002. Proceedings of the annual reliability and maintainability symposium, 2002.

MALKA, Y.; ZIV, A. Design reliability - estimation through statistical analysis of bug discovery data. In: DESIGN AUTOMATION CONFERENCE, 10-13 February, 1998. Design Automation Conference Proceedings, p. 644649, 1998.

MARTZ, H. F.; WALLER, R. A. Bayesian reliability analysis. Krieger Publishing, Malabar, Florida, 1991.

MEEKER, W. Q.; ESCOBAR, L. A. Statistical methods for reliability data. John Wiley \& Sons, New York, 1998.

MOURAD, S.; FUJIWARA, H. Design for Reliability. In: INSTRUMENTATION AND MEASUREMENT TECHNOLOGY CONFERENCE, Como, Italy. Proceedings of the instrumentation and measurement technology conference, p. 2033-2035, 2004.

MUSA, J. D.; OKUMOTO, K. A logarithmic Poisson execution time model for software reliability measurement. In: SEVENTH INTERNATIONAL CONFERENCE ON SOFTWARE ENGINEERING, 22-24 June, 1984, Rockville, Maryland, USA. Proceedings of the seventh international conference on software engineering, p. 230-238, 1984. 
NELSON, W. Applied life data analysis. John Wiley and Sons, 1982.

Weibull analysis of reliability data with few or no failures. Journal of Quality \& Technology, v. 17, n. 3, p. 140-146, 1985.

PATTERSON-HINE, F. A.; IVERSON, D. L. An integrated approach to system design, reliability, and diagnosis. In: ${ }^{\text {th }}$ DIGITAL AVIONICS SYSTEMS CONFERENCE, IEEE/AIAA/NASA, 15-18 October, Virginia Beach, USA. Proceedings of the $\mathbf{9}^{\text {th }}$ Digital Avionics Systems Conference, p. 480-487, 1990.

PRIEST, W. J.; SÁNCHEZ, J. M. Product development and design for manufacturing. Second Edition, Marcel Dekker, New York, 2001.
RAUSAND, M.; HOYLAND, A. System reliability theory. Second Edition, John Wiley \& Sons, New York, 2004.

SIU, N. O.; KELLY, D. L. Bayesian parameter estimation in probabilistic risk assessment. Reliability Engineering \& System Safety. v. 62, p. 89-116, 1998.

TELECORDIA TECHNOLOGIES. Reliability prediction procedure for electronic equipment, SR-332, Issue 1, May, 2001.

TIAN, X. Design-for-reliability and implementation on power converters. In: ANNUAL RELIABILITY AND MAINTAINABILITY SYMPOSIUM, 24-27 January, Alexandria, USA, 2005. Proceedings of the annual reliability and maintainability symposium, p. 89-95, 2005.

\title{
RELIABILITY ASSESSMENT OF PRODUCTS UNDER DEVELOPMENT
}

\begin{abstract}
This paper presents a method to assess the reliability of products under development. The proposed methodology allows for the use of various sources of information commonly found in the development stages of a product, such as field or warranty data (failure rates), test data and subjective evidence (engineers/operators'opinions about the impact of design changes on the product's reliability). The method also allows for the incorporation of evidence of previous revisions of the same product or even information about products that are only similar to the one under investigation. The proposed methodology is illustrated through an example of the reliability assessment of high power X-ray tubes, which confirms that the reliability assessment of a new design before the actual testing of units containing the proposed design modifications offers the manufacturer a relevant source of information upon which to base decisions about the effective implementation of the design modifications.
\end{abstract}

Keywords: reliability, product development, bayes' theorem. 
